\section{ESTRATEGIA DE PROMOCIÓN PARA POSICIONAR UNA EMPRESA EN LA CIUDAD DE POTOSÍ}

\author{
PROMOTION STRATEGY TO POSITION A COMPANY IN THE \\ CITY FROM POTOSÍ
}

\author{
Rita Fabiola Gómez Tapia
}

Artículo recibido marzo 2019 | Arbitrado abril 2019 | Publicado 01 de julio 2019

\section{Resumen}

La Cristalería "Sissy" es una empresa potosina dedicada al servicio de flete y alquiler de cristalería y vajilla. El propósito de estudio que se desarrollo fue elaborar una estrategia de promoción para posicionar la Cristalería "Sissy" en la ciudad de Potosí, donde se utilizaron como métodos teóricos los siguientes: Análisis-síntesis, inductivo-deductivo, histórico-lógico y comparativo; entre los métodos empíricos se emplearon: la entrevista, encuesta y la observación. Los resultados del diagnóstico permitieron elaborar una estrategia de promoción basada en tácticas de la mezcla promocional (promoción de ventas, publicidad, relaciones públicas, venta personal y marketing directo) como ser: cupones, descuentos, premios de fidelidad, televisión, radio, periódico, internet, publicaciones, patrocinios, publicidad, capacitación a empleados y venta por catálogo. Como conclusión final se espera que la aplicación de la propuesta ayude a fortalecer el posicionamiento de la Cristalería "Sissy".

Palabras clave: estrategias de promoción; publicidad; relaciones públicas

\section{Abstract}

The "Sissy" Glassware is a potosina company dedicated to the service of freight and rental of glassware and dishes, the study aimed to develop a promotional strategy to position the "Sissy" Glassware in the city of Potosí, where they were used as theoretical methods the following: Analysissynthesis, inductive-deductive, historical-logical and comparative; Among the empirical methods were used: the interview, survey and observation. The results of the diagnosis allowed developing a promotion strategy based on tactics of the promotional mix (sales promotion, advertising, public relations, personal sales and direct marketing) such as: coupons, discounts, loyalty awards, television, radio, newspaper, internet, publications, sponsorships, publicity, and employee training and catalog sales. As a final conclusion, the application of the proposal is expected to help strengthen the positioning of the "Sissy" Glassware.

Key words: promotion strategies; advertising; public relations

\section{Rita Fabiola Gómez Tapia}

rita_gt93@hotmail.com

Universidad Privada Domingo Savio, Bolivia

Ingeniero Comercial. Egresada de la Universidad Privada Domingo Savio. Capacitación de Principios de Autosuficiencia Aplicada, realizado el 29 de mayo de 2016. Taller "Ley SAFCO 1178 Gobernabilidad" como se diseñan y gestionan las políticas en el Estado Plurinacional de Bolivia, realizado el 5 y 6 de mayo de 2017 en la ciudad de Cochabamba. Congreso Latinoamericano de Ciencias Económicas, Financieras, Contables, Comerciales y Administrativas, realizado el 5 y 6 de mayo del 2017 en la ciudad de Cochabamba "el futuro de la publicidad" realizado el 19 de mayo del 2017 en la ciudad de Sucre "Neuromarketing" realizado el 20 de mayo del 2017 en la ciudad de Sucre. 


\section{INTRODUCCIÓN}

Todo emprendimiento tiene una meta, en la mayoría de los casos busca beneficios económicos, y muchas organizaciones utilizan algunas estrategias para alcanzar ese fin, por ejemplo el Marketing, que ayuda a identificar $y$ satisfacer las necesidades $y$ deseos de los consumidores, ya que tiene la función principal de desarrollar y mejorar los productos o servicios que van dirigidos específicamente de acuerdo a los intereses del cliente, además sirve como una ventaja frente a la competencia ya que existen nuevos gustos y exigencias en el mercado, $y$ porque aparecen nuevos segmentos que antes se desconocían.

Para este hecho es necesario utilizar el Marketing Mix que consiste en mezclar las variables controlables por parte de la empresa como son las $4 \mathrm{P}^{\prime} \mathrm{s}$, es decir: el Producto, el Precio, la Plaza y por último la Promoción; estas variables siempre estarán relacionadas entre sí, y dependerán unas de otras para así generar una oferta de marketing eficaz.

Cabe recalcar que en un mercado saturado con muchos productos o servicios ofreciendo beneficios similares, un buen posicionamiento hace a una marca o producto resaltar del resto, ofreciéndole la posibilidad de cobrar un mayor precio $y$ mantener lejos a la competencia en términos de resultados. Además permite a la empresa, superar malas temporadas más fácilmente, ofrece mayor flexibilidad en términos de extensiones, cambios, distribución y publicidad.

Por otra parte, por medio del posicionamiento, una empresa desconocida, puede satisfacer con eficacia la necesidad del consumidor, al crear una imagen mental del producto o servicio con sus diferentes características, consiguiendo que éste tenga una percepción positiva y le dé significado al mismo. Pero para que sucedan estos hechos, se debe construir una comunicación activa de los atributos, beneficios o valores distintivos del producto, servicio o marca, al grupo objetivo previamente seleccionado en base a la estrategia empresarial. El posicionamiento es ampliamente codiciado por todo tipo de organizaciones tanto privadas como públicas, tal es el caso de las cristalerías que se dedican al flete de sus productos.

Las empresas Menaje Rent y Enjoy ubicadas en Perú y Argentina respectivamente, que se dedican al flete $y$ alquiler de cristalería, se encuentran bien posicionadas en el mercado gracias a su estrategia de marketing, pero en Bolivia, más propiamente en la Ciudad de Potosí, no existen empresas dedicadas al flete o alquiler de cristalería que estén posicionadas en el mercado.

Tal es el caso de La Cristalería "SISSY", una empresa potosina, que se creó con el propósito de generar ingresos para el hogar; es una empresa Unipersonal dedicada al flete y alquiler de Cristalería y Vajilla, tiene 29 años de vida en este medio, actualmente tiene más de 20 productos para fletar $y$ alquilar, para lo cual cuenta con: Champaneras, Cocteleras, Cerveceros, Chuflayeras, Vineras, Wisqueras, Gelatineras, Platos, Cubiertos, Jarras, Tazas, Platillos, Paneros, Lecheras, Azucareras, Hieleras, Pinzas, Fuentes de Vidrio, Soperas, Charolas, Floreros, etc.

Si bien dicha empresa se caracteriza por la limpieza y variedad de productos, no todos la conocen, debido a que no existen estrategias que ayuden a captar el interés de las personas para lograr el posicionamiento deseado en el mercado potosino. 
Por tanto, el presente trabajo de investigación se enfocó en el posicionamiento de la Cristalería "Sissy" en la ciudad de Potosí, para lo cual se logró identificar las siguientes dificultades: Gran parte de la población potosina no conoce la cristalería "Sissy" lo cual perjudica en las labores que desempeña la misma y no logra obtener sus objetivos deseados; Existe confusión por parte de la población potosina al momento de tener contacto con la Cristalería "Sissy" ya que no tienen bien claro si la misma vende productos o se dedica al flete de los mismos; Muchas personas que no conocen los productos que ofrece la Cristalería "Sissy" prefieren buscar salones de eventos que ofrecen su propia cristalería, aun cuando éstos no tengan lo que el cliente pide; La población al tener desconocimiento de una empresa que realiza flete de cristalería en algunos casos optan por comprar estos productos para distintos eventos sociales; Se realizó una prueba piloto, en la Universidad Privada Domingo Savio, a 30 personas, hombres y mujeres entre las edades de 18 a 40 años. Los resultados de la prueba piloto nos dieron a entender que la Cristalería "Sissy no es conocida en el mercado potosino, un $100 \%$ de la población encuestada respondió que desconocen dicha empresa y no saben qué servicios ofrece, por nombrar alguna de ellas.

Analizando todas estas dificultades encontradas en dicha empresa, se pudo determinar el siguiente problema científico: ¿Cómo posicionar la Cristalería "Sissy" en la ciudad de Potosí?

Así también se determina que el objeto de estudio es: el posicionamiento, el campo de acción es el Posicionamiento de la Cristalería "Sissy" en la ciudad de Potosí, en concordancia se propone como objetivo general: "Elaborar una estrategia de promoción para posicionar la Cristalería "Sissy" en la ciudad de Potosí."

De esta manera, para una mejor orientación en el proceso de investigación, se planteó las siguientes preguntas científicas:

P.1.: ¿Cuáles son los fundamentos teóricos del posicionamiento y de la estrategia de promoción?

P.2.: ¿Cuál es la situación actual de la Cristalería "Sissy" con respecto a su posicionamiento en la ciudad de Potosí?

P.3.: ¿Cuáles son las tácticas más apropiadas para la elaboración de una estrategia de promoción para posicionar la Cristalería "Sissy" en la ciudad de Potosí?

Las tareas científicas establecidas según las preguntas anteriores para dar respuesta a la problemática planteada en el estudio que se llevó a cabo en la Cristalería Sissy estuvieron orientadas al establecimiento de los fundamentos teóricos del posicionamiento y de la estrategia; como la caracterización del estado actual del posicionamiento de la cristalería; para finalizar se elaboraron las tácticas más apropiadas de una estrategias de promoción para posicionar la cristalería "Sissy" en la ciudad de Potosí.

Sobre la justificación de la presente investigación se dice que la aproximación a la situación en la que se ve la Cristalería "Sissy" en relación al posicionamiento, no está posicionada en el mercado potosino, pues la mayoría de la población desconoce la existencia de la Cristalería. Para cualquier empresa es muy importante estar posicionada en el mercado, y sobre todo en la mente de los consumidores o clientes, 
porque permite el desarrollo y crecimiento de la empresa en cualquier aspecto. El posicionamiento tiene que ser una parte fundamental para el éxito en cualquier organización, es por eso que se esfuerza y hace todo lo posible por ser el producto o servicio favorito de las personas, haciendo que su producto o servicio sea el más utilizado y consumido por las personas y de esta manera se mantenga por largo tiempo en el mercado y obtenga ganancias.

Esta investigación contribuye a un aporte para la comunidad científica para las consultas de los estudiantes y docentes y para empresas del mismo rubro.

\section{MÉTODO}

La metodología empleada en este estudio fue de bajo el paradigma cuantitativa porque el proceso se basó en los datos numéricos y estadísticos, los cuales se obtuvo de los datos estadísticos del I.N.E. y de la recolección de datos mediante la encuesta que se realizó en la investigación, propiamente en el diagnóstico. Y cualitativa porque se realizó la interpretación o valoración de los resultados de la recolección de datos mediante la entrevista y la guía de observación.

Además, el tipo de investigación fue descriptiva porque a través de la bibliografía y los datos recabados sobre el tema se obtuvo el conocimiento de las propiedades, las características importantes y la información necesaria sobre el posicionamiento y la estrategia de promoción, que permitió trabajar a base de esos hechos reales y de una manera más precisa y específica. El plan para obtener la información que se desea para el diseño de investigación fue por medio de una recopilación documental en base a referencias bibliográficas, también se realizó un trabajo de campo utilizando los instrumentos de recolección de información como la encuesta, la entrevista y la guía de observación que se redactó en el diagnóstico.

Los métodos teóricos que se utilizaron fueron análisis - síntesis, ya que este método permitió analizar el posicionamiento descomponiendo en sus partes más importantes para llegar a conocer cada elemento, la síntesis ayudó a unir cada una de las partes previamente analizadas con respecto al posicionamiento, lo que ayudó a reconocer las características más importantes de cada componente, coadyuvando así en el marco teórico y en el diagnóstico, el método inductivo - deductivo porque se partió de circunstancias particulares sobre el posicionamiento, desde un diagnóstico el cual ayudó a detectar el problema. Posteriormente se realizó la deducción sobre el posicionamiento en el marco teórico y se generalizó la información obtenida del diagnóstico hacia la propuesta.

Los métodos empíricos aplicados fueron la entrevista más propiamente la entrevista estructurada en base a la operacionalización de la variable dependiente, dirigida a la propietaria y al personal de la Cristalería "Sissy", con el objetivo de obtener la información necesaria y precisa sobre el posicionamiento de dicha Cristalería en la ciudad de Potosí, plasmada en el diagnóstico. Se aplicó una encuesta por medio de un cuestionario con preguntas cerradas a toda la población interesada de la ciudad de Potosí. Con el propósito de tener la información necesaria y precisa sobre el posicionamiento de la Cristalería "Sissy".

Para la presente investigación se tomó en cuenta como población a las personas hombres y mujeres comprendidas entre los 18 y 65 años de edad, residentes de la ciudad 
de Potosí. Se tomó en cuenta a mujeres porque son las que siempre están organizando eventos como: cumpleaños, quince años, matrimonios, bautizos, fiestas, etc. y cuando los hijos (menores de edad) necesiten el servicio de una cristalería siempre recurren a la madre, también se tomó en cuenta a los hombres puesto que muchos tienen su propio negocio y requieren el servicio de una cristalería, Así mismo se tomó en cuenta de 18 a 65 años de edad ya que tienen poder adquisitivo y poder de decisión, conformando este segmento un total de 108.424 personas, según datos recabados del I.N.E.

Una vez realizado el cálculo de la muestra, aplicando la fórmula de las poblaciones finitas, se tuvo como resultado realizar la encuesta a 383 personas; el tipo de muestreo que se adoptó para realizar esta investigación fue probabilístico aleatorio simple, ya que todos tienen la misma posibilidad de ser encuestados.

\section{RESULTADOS Y DISCUSION}

De acuerdo al estudio que se llevó a cabo como resultado se elaboraron las tácticas de una estrategia de promoción para posicionar la Cristalería "Sissy" en la ciudad de Potosí

Para ello, se da a conocer los elementos apropiados de una estrategia de promoción para posicionar la Cristalería "Sissy" diseñada de acuerdo a las necesidades que presenta dicha empresa. Se recogió información del personal que trabaja en la Cristalería "Sissy", así como de la población potosina a quienes se aplicó la encuesta, y con la misma se estableció un diagnóstico de la situación. Descubriendo así la falencia y dificultades que presentan en cuanto al posicionamiento. (Ver figura 1)

\section{Estructura de la propuesta}

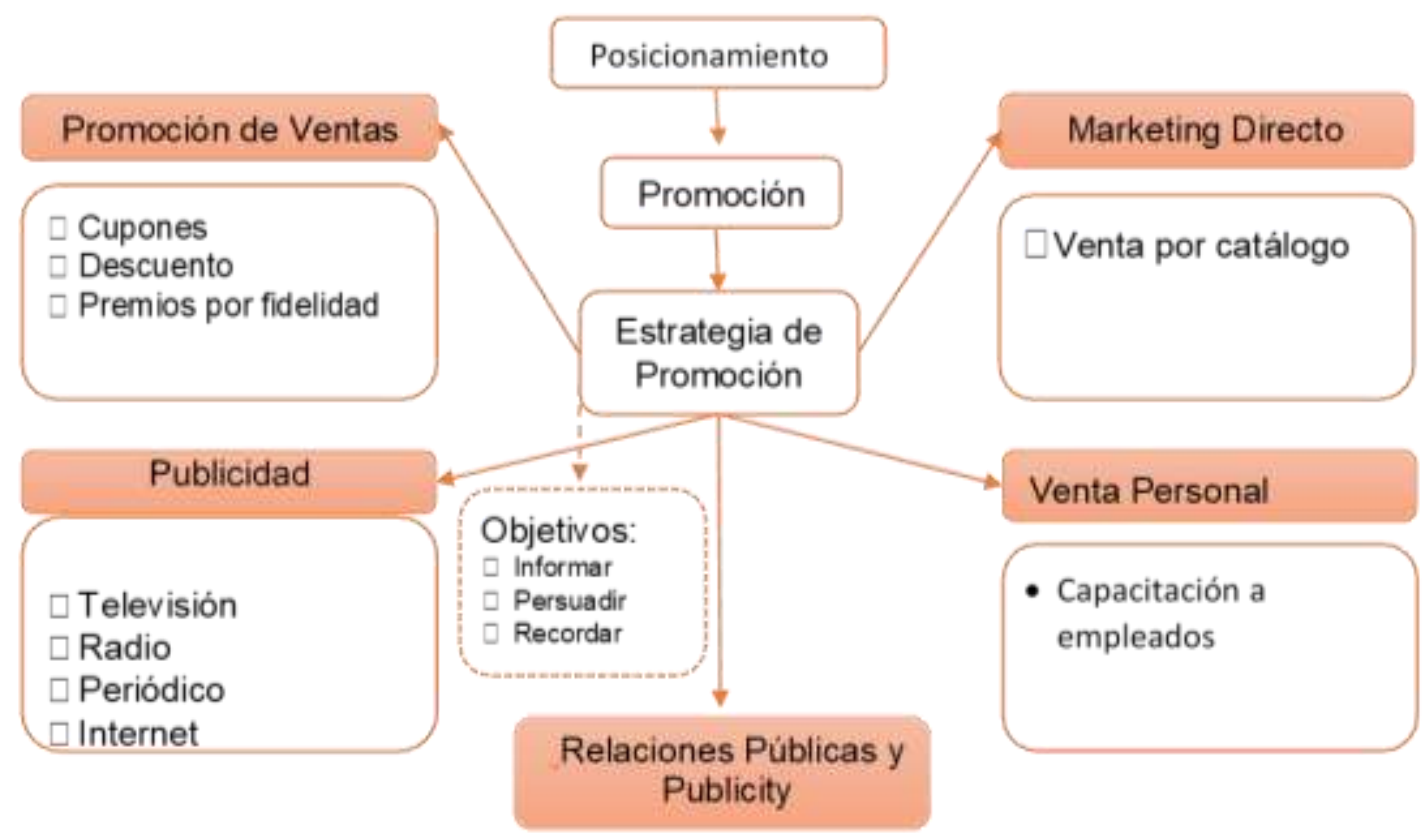

Figura 1. Estructura de la propuesta. Adaptado de "Fundamentos de marketing", Monferrer, D., 2013, pp.155171, Recuperado de http://repositori.uji.es/xmlui/bitstream/handle/10234/49394/s74.pdf 


\section{Elaboración de la propuesta}

Se definió una propuesta basada en la elaboración de una estrategia de promoción para posicionar la Cristalería "Sissy" afín de ayudar a la empresa a darse a conocer en la ciudad de Potosí.

\section{Mercado meta}

Estuvo enfocada para llegar a la mente de personas, hombres y mujeres de 18 a 65 años de edad de la ciudad de potosí, ya que los mismos son los consumidores con mayor tendencia a requerir los productos de la Cristalería "Sissy".

\section{Tácticas de la estrategia de promoción}

A continuación se presentan las tácticas de las diferentes variables de la mezcla promocional que formó parte de la estrategia de marketing para la Cristalería "Sissy".

\section{Tácticas de Promoción de Ventas}

\section{Cupones}

Con el fin de aumentar la compra del servicio y combatir la actividad promocional de los competidores, se utilizaron vales certificados que proporcionaban a los compradores un ahorro en la cantidad a pagar cuando se fletaban o alquilan la cristalería o vajilla.

Se otorgaron los vales certificados a todas las personas que realizaban el flete $o$ alquiler de cristalería o vajilla por un monto mayor a $100 \mathrm{Bs}$, de la siguiente manera:

- Por el flete de 100 Bs un vale certificado de 10 Bs

- Por el flete de 200 Bs un vale certificado de 20 Bs

- Por el flete de 300 Bs un vale certificado de 30 Bs

- Por el flete de 400 Bs un vale certificado de 40 Bs

- Por el flete de 500 Bs un vale certificado de $50 \mathrm{Bs}$

Una vez que el cliente usase el vale certificado, se devuelve el cupón al personal. A continuación se presenta el diseño de los cupones $o$ vales certificados que se otorgaron, diferenciando cada cupón por colores de acuerdo al precio: 


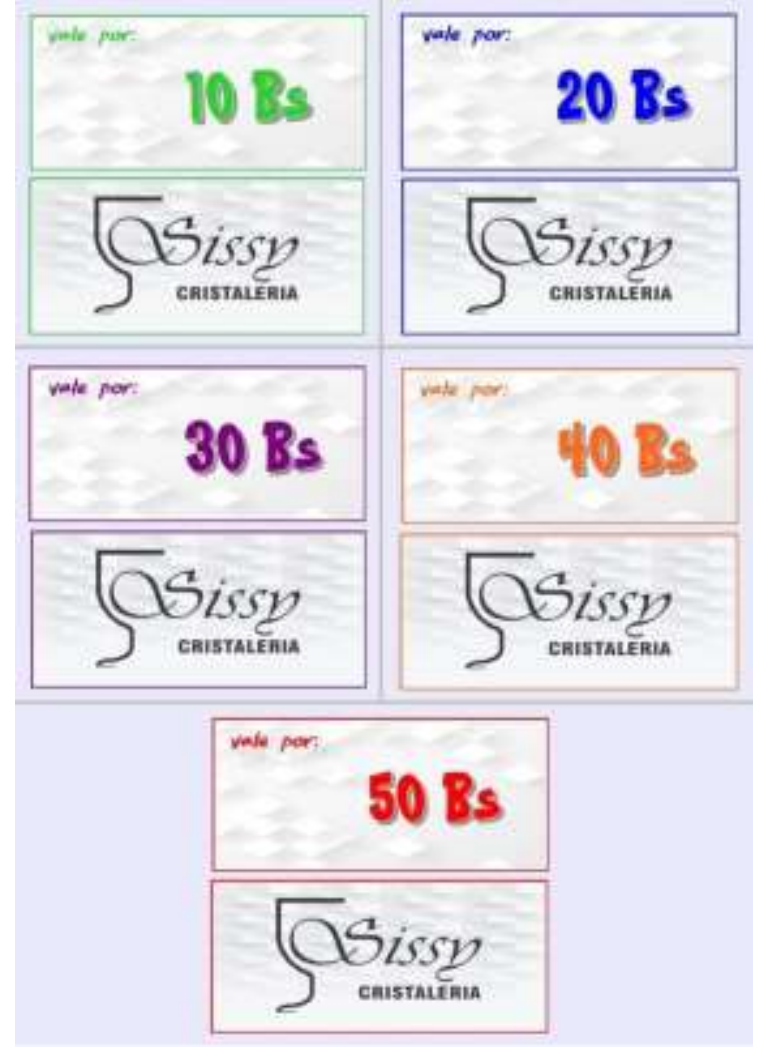

Figura 2. Diseño de cupones o vales certificados

Tabla 1. Costo cupones (Expresado en Bolivianos)

\begin{tabular}{|c|c|c|c|c|c|c|}
\hline MES & CUPONES & $\begin{array}{c}\text { TOTAL } \\
\text { UNIDADES }\end{array}$ & $\begin{array}{l}\text { IMPRESIÓN/ } \\
\text { UNIDAD }\end{array}$ & $\begin{array}{c}\text { PLASTIFICADO/ } \\
\text { UNIDAD }\end{array}$ & COSTO TOTAL & \\
\hline \multirow{5}{*}{ Enero } & de 10 Bs. & 3 & 1 & 3 & 12 & \\
\hline & de 20 Bs. & 3 & 1 & 3 & 12 & \\
\hline & de $30 \mathrm{Bs}$. & 3 & 1 & 3 & 12 & \\
\hline & de $40 \mathrm{Bs}$. & 3 & 1 & 3 & 12 & \\
\hline & de 50 Bs. & 3 & 1 & 3 & 12 & \\
\hline \multirow{8}{*}{ Junio } & & & & Total cupones & 60 & 60 \\
\hline & de 10 Bs. & 3 & 1 & 3 & 12 & \\
\hline & de 20 Bs. & 3 & 1 & 3 & 12 & \\
\hline & de 30 Bs. & 3 & 1 & 3 & 12 & \\
\hline & de 40 Bs. & 3 & 1 & 3 & 12 & \\
\hline & de 50 Bs. & 3 & 1 & 3 & 12 & \\
\hline & & & & Total cupones & 60 & 60 \\
\hline & \multicolumn{5}{|c|}{ Costo total cupones } & 120 \\
\hline
\end{tabular}

Nota: Elaboración propia en base a los costos estimados 


\section{Descuento}

Para persuadir a los clientes a comprar más seguido este servicio, se realizó una reducción directa sobre los precios de los productos y la garantía del flete durante un periodo de tiempo.

Normalmente la garantía es entre 500 a 700 bolivianos pero si el cliente viene de manera consecuente se hará una rebaja en su garantía, siempre y cuando sea en efectivo. A continuación el detalle:

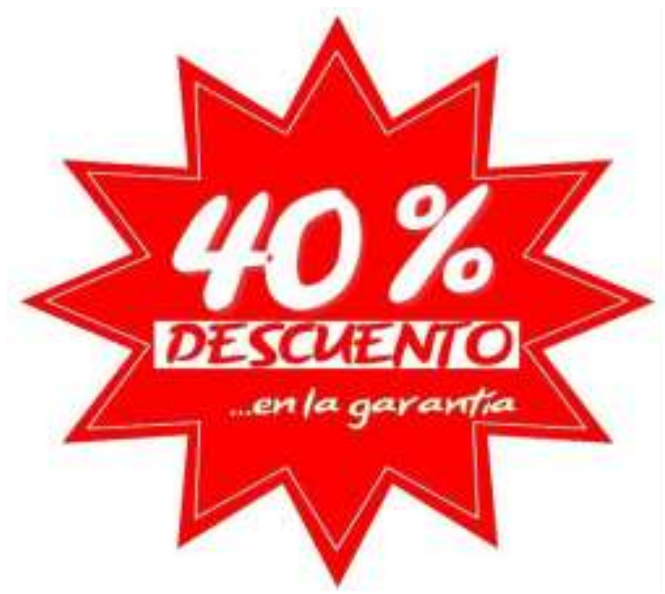

- El descuento consistirá en el $40 \%$ del total de la garantía, es decir, con este descuento la garantía será 300 a 420 Bs.

- En cuanto al precio, normalmente es de 38 bolivianos, se hará un descuento del $8 \%$, con este descuento el precio será $35 \mathrm{Bs}$.

A continuación se presenta el diseño de los anuncios de descuento a la vista del cliente:

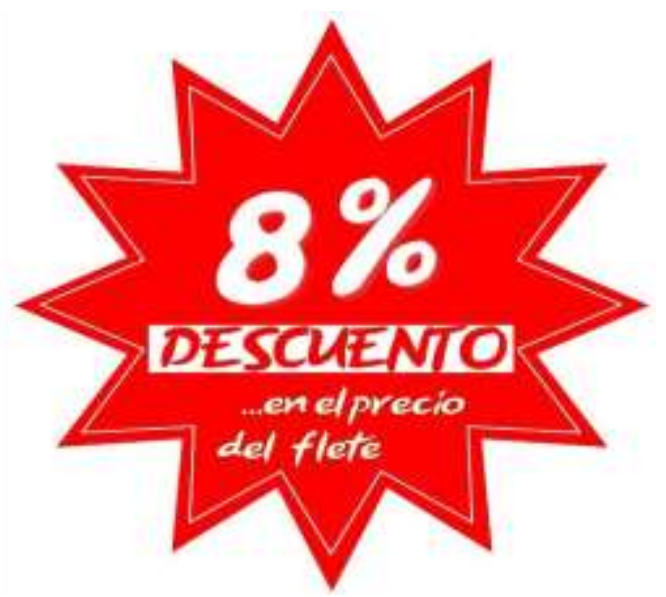

Figura 3. Diseño de anuncio de descuento

Tabla 2. Costo Anuncio de descuento (Expresado en Bolivianos)

\begin{tabular}{llcccc}
\hline \multirow{2}{*}{ MES } & DESCUENTO & $\begin{array}{c}\text { TOTAL } \\
\text { UNIDADES }\end{array}$ & $\begin{array}{c}\text { IMPRESIÓN/ } \\
\text { UNIDAD }\end{array}$ & $\begin{array}{c}\text { PLASTIFICADO/ } \\
\text { UNIDAD }\end{array}$ & $\begin{array}{c}\text { COSTO } \\
\text { TOTAL }\end{array}$ \\
\hline \multirow{2}{*}{ Enero } & Anuncio del 40\% & 1 & 1 & 7 & 8 \\
& Anuncio del $8 \%$ & 1 & 1 & 7 & 8 \\
\hline & & \multicolumn{2}{c}{ Costo total anuncio de Descuento } & $\mathbf{1 6}$ \\
\hline
\end{tabular}

Nota: Elaboración propia en base a los costos estimados 


\section{Premios por fidelidad}

Con el objetivo de recompensar a los clientes fieles, se otorgará premios a fin de año, por la compra habitual de los servicios que realicen durante todo el año.

Los premios consisten en adornos navideños con el logo de la cristalería, estos adornos serán hechos de aquellos artículos o productos que lleguen a desportillarse, rajarse o maltratarse, y adornos o pintura que la administradora y el personal tienen a disposición, es decir, se realizara con material reciclado, por lo tanto no se incurrirá en ningún gasto. A continuación, un ejemplo de los premios que se otorgará a los clientes fieles hecho por la administradora.

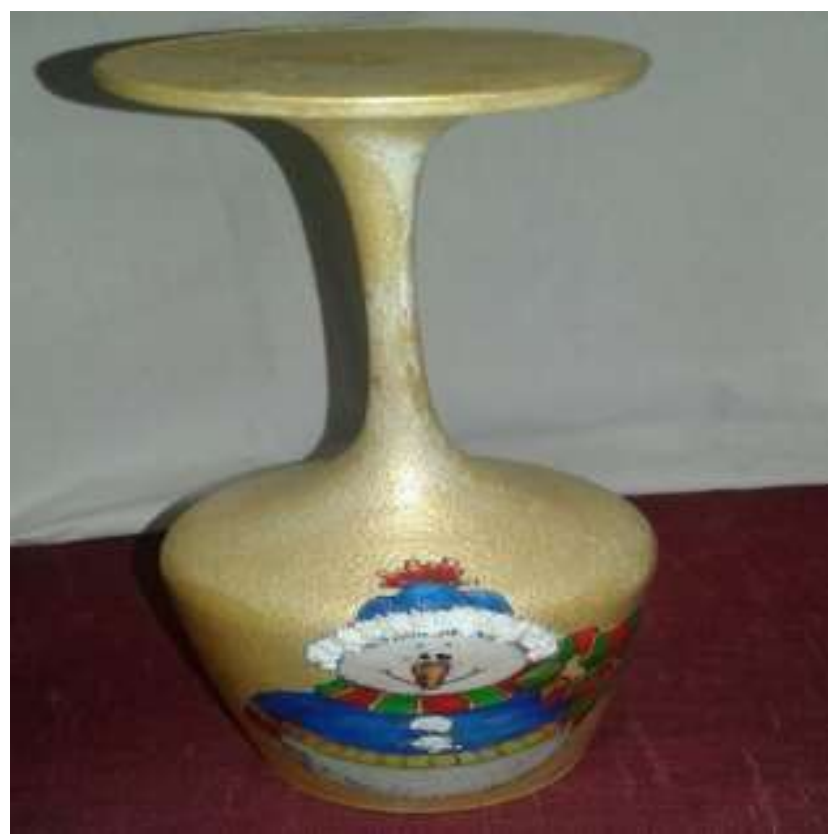

Figura 4. Premios por fidelidad. Fotografías tomadas de los adornos navideños realizados por la administradora

\section{Tácticas de Publicidad}

\section{Televisión}

Se realizará un spot publicitario en televisión, ya que combina visión, contenido y movimiento por lo que tiene alto poder de atracción y elevada audiencia.

Al obtener los resultados de la encuesta se elige como canal televisivo óptimo la Red Uno al ser el medio de mayor preferencia.
Haciendo uso del "paquete especial", el spot publicitario de la Cristalería "Sissy" se transmitirá de lunes a viernes a las 9:00, $10: 00,16: 00$ y $20: 00$ horas; sábado a las 9:00, $13: 00,19: 00$ y 23:00 horas y domingo a las $12: 00,13: 00,14: 00$ y 22:00 horas, durante los meses de mayo, septiembre y diciembre, ya que son los meses donde hay más eventos que el resto del año. 
Tabla 3. Diseño del spot publicitario

VIDEO
Imagen del logotipo
Información del
servicio

Características
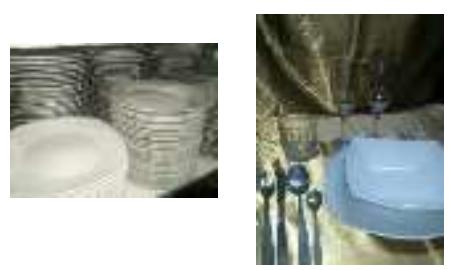

Datos de la empresa
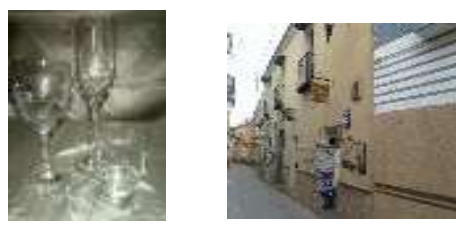

Mensaje final y logotipo con los contactos

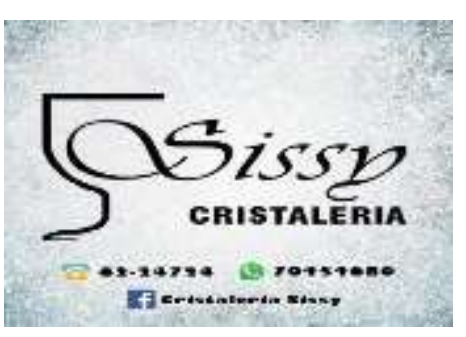

\section{AUDIO}

Cristalería Sissy un servicio de calidad

Somos una empresa dedicada al servicio de flete y alquiler de cristalería y vajilla. Con más de 20 años de experiencia.

En la Cristalería Sissy encontraras más de 20 productos de la más alta calidad y reconocidas marcas, productos de diferentes modelos a su gusto y elección.

Nos encontramos en la calle sucre \# 56 , zona central

El cliente es nuestra prioridad, por ello le brindaremos un servicio de calidad

El spot publicitario ira acompañado con el audio musical de fondo "Life's happy journey"

Tabla 4. Presupuesto del spot publicitario

\begin{tabular}{cccccc}
\hline \multirow{2}{*}{ MES } & \multicolumn{3}{c}{$\mathbf{N}^{\circ}$ DE PASES EN LA RED UNO “Paquete especial” } & \multirow{2}{c}{ COSTO } \\
\cline { 2 - 4 } & $\mathbf{1}^{\circ}$ SEMANA & $\mathbf{2}^{\circ}$ SEMANA & $\mathbf{3}^{\circ}$ SEMANA & $\mathbf{4}^{\circ}$ SEMANA & TOTAL (Bs) \\
\hline Mayo & 28 & 28 & 28 & 28 & 1600 \\
Septiembre & 28 & 28 & 28 & 28 & 1600 \\
Diciembre & 28 & 28 & 28 & 28 & 1600 \\
\hline
\end{tabular}

Nota: Elaboración propia en base a los costos publicitarios proporcionados por la Red Uno. 


\section{Radio}

Por la utilización masiva y propiamente por las instituciones se realizará un jingle que se difundirá por la Radiodifusora Kollasuyo, ya que de acuerdo a los resultados obtenidos de la encuesta se elige como emisora de mayor frecuencia. El jingle durará 30 segundos y tendrá 3 pases al día en el programa Cambalache durante los meses de febrero, abril, agosto y noviembre, aprovechando los días festivos y fechas culturales.

Tabla 5. Diseño del jingle

\begin{tabular}{|c|c|c|}
\hline MÚSICA & ESTRUCTURA & AUDIO \\
\hline \multirow{5}{*}{ Life's happy journey } & Slogan & $\begin{array}{l}\text { Cristalería Sissy un servicio de } \\
\text { calidad }\end{array}$ \\
\hline & Información del servicio & $\begin{array}{l}\text { Somos una empresa dedicada al } \\
\text { servicio de flete y alquiler de } \\
\text { cristalería y vajilla. Con más de } 20 \\
\text { años de experiencia. }\end{array}$ \\
\hline & & $\begin{array}{l}\text { En la Cristalería Sissy encontraras } \\
\text { más de } 20 \text { productos de la más alta }\end{array}$ \\
\hline & Características del servicio & $\begin{array}{l}\text { calidad y reconocidas marcas, } \\
\text { productos de diferentes modelos a } \\
\text { su gusto y elección }\end{array}$ \\
\hline & Datos de la empresa & $\begin{array}{l}\text { Nos encontramos en la calle sucre } \\
\text { \# 56, zona central }\end{array}$ \\
\hline
\end{tabular}

Tabla 6. Presupuesto del jingle

\begin{tabular}{lccccc}
\hline \multirow{2}{*}{ MES } & \multicolumn{2}{c}{$\mathbf{N}^{\circ}$ DE PASES EN LA RADIO KOLLASUYO “Cambalache” } & \multicolumn{2}{c}{$\begin{array}{c}\text { COSTO TOTAL } \\
\text { (Bs) }\end{array}$} \\
\cline { 2 - 5 } & $\mathbf{1}^{\circ}$ SEMANA & $\mathbf{2}^{\circ}$ SEMANA & $\mathbf{3}^{\circ}$ SEMANA & $\mathbf{4}^{\circ}$ SEMANA & 500 \\
\hline Febrero & 21 & 21 & 21 & 21 & 500 \\
Abril & 21 & 21 & 21 & 21 & 500 \\
Agosto & 21 & 21 & 21 & 21 & 500 \\
Noviembre & 21 & 21 & 21 & 21 & $\mathbf{2 0 0 0}$ \\
\hline
\end{tabular}

Nota: Elaboración propia en base a los costos publicitarios proporcionados por la radio Kollasuyo

\section{Periódico}

Pensando en aquellas personas mayores y aquellas que siempre están buscando información en la prensa diaria, se elige el periódico El Potosí el cual es el medio con más frecuencia según la encuesta realizada.
Por tanto se publicará un anuncio en el Baúl a full color, del tamaño de $3 \times 2(11 \mathrm{~cm} x$ $6,7 \mathrm{~cm}$ ), durante los meses de marzo, junio y octubre. 
A continuación se presenta el diseño del anuncio publicitario en el periódico El Potosí con el logo de la cristalería, el servicio que ofrece y los datos de la empresa.

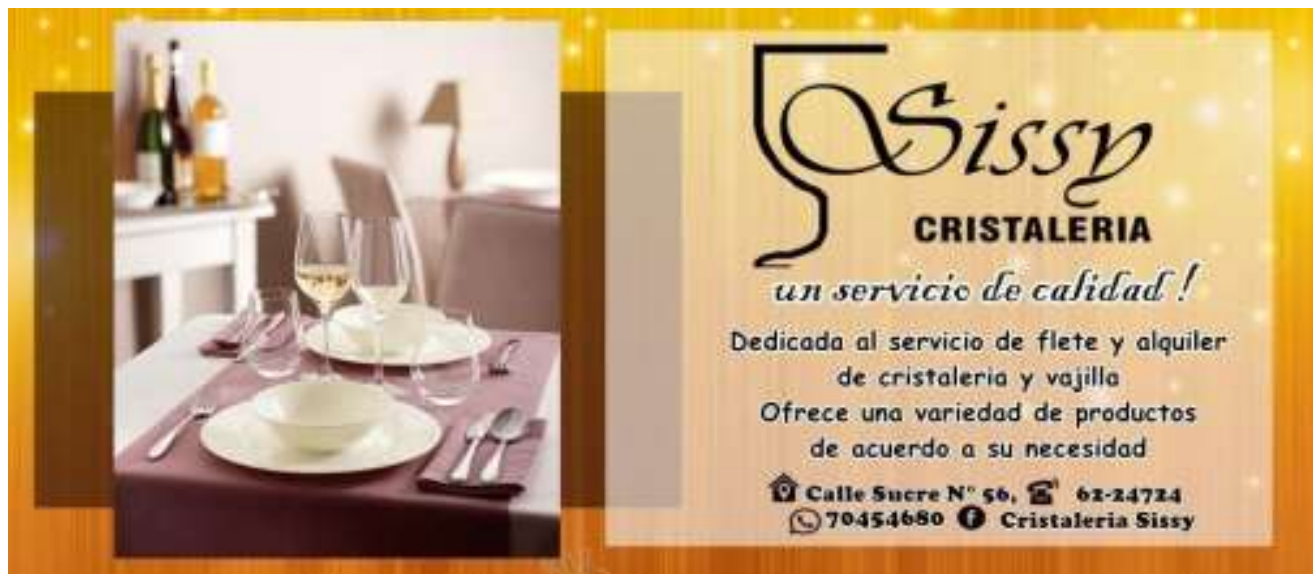

Figura 5. Diseño de publicidad en el periódico

Tabla 6. Presupuesto publicidad en el periódico (expresado en bolivianos)

\begin{tabular}{cccc}
\hline MES & PUBLICACIONES & COSTO MENSUAL & COSTO TOTAL \\
\hline Marzo & 30 publicaciones & 600 & 600 \\
Junio & 30 publicaciones & 600 & 600 \\
Octubre & 30 publicaciones & 600 & 600 \\
\hline & Total publicidad en el periódico & $\mathbf{1 8 0 0}$
\end{tabular}

Nota: Elaboración propia en base a los costos publicitarios proporcionados por el periódico El Potosí

\section{Internet}

Gracias al gran alcance que se tiene por medio de internet, la cristalería puede promocionar el servicio y tener una mejor relación con el público objetivo, y aprovechando que la Cristalería "Sissy" ya cuenta con $\mathrm{Wi}-\mathrm{Fi}$ se procede a realizar la creación de cuentas, en Facebook, Instagram y WhatsApp.

- Cuenta en Facebook

La cuenta de Facebook estará activa y pendiente a cualquier solicitud, opiniones o sugerencias que el mercado potencial haga, así también, se tendrá actualizaciones frecuentes, fotos, información o anuncios que ayude a promocionar el servicio, hacerse conocer y captar clientes, especialmente gente joven y estudiantes que siempre están conectados y pendientes a esta red social.

Cabe recalcar que según los resultados de la encuesta, el $87 \%$ de la población encuestada mostró interés en conocer la Cristalería "Sissy" dejando su nombre y cuenta de Facebook, a quienes se les puede hacer llegar la invitación o solicitud de parte de la cristalería 


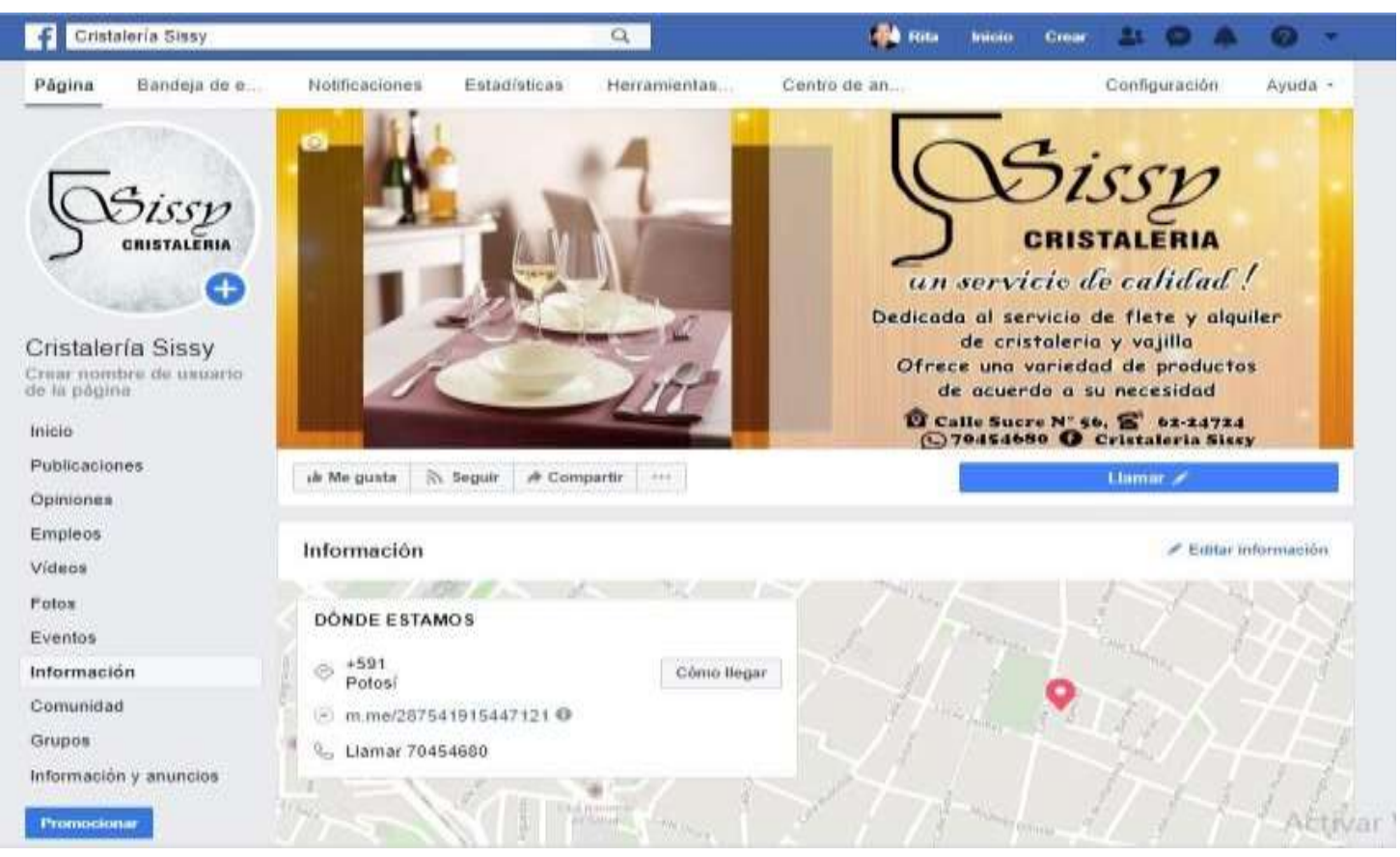

Figura 6. Diseño de página de Facebook “Cristalería Sissy”. Extraído de https://www.facebook.com/Cristaler\%C3\%ADa-Sissy 287541915447121/?modal=admin_todo_tour

Esta página permitirá promocionar el servicio. La información de la Cristalería en esta página hace que las personas interesadas puedan llamar y preguntar sobre el servicio que requiere.

- Cuenta en Instagram

Instagram es un sitio donde se comparte especialmente fotos y videos, por lo tanto, la Cristalería "Sissy", se ocupará de subir fotos o videos creativos, llamativos y agradables a la vista, de manera que estas fotos digan más que mil palabras, dirigidas a personas que requieren un servicio de buena calidad. 


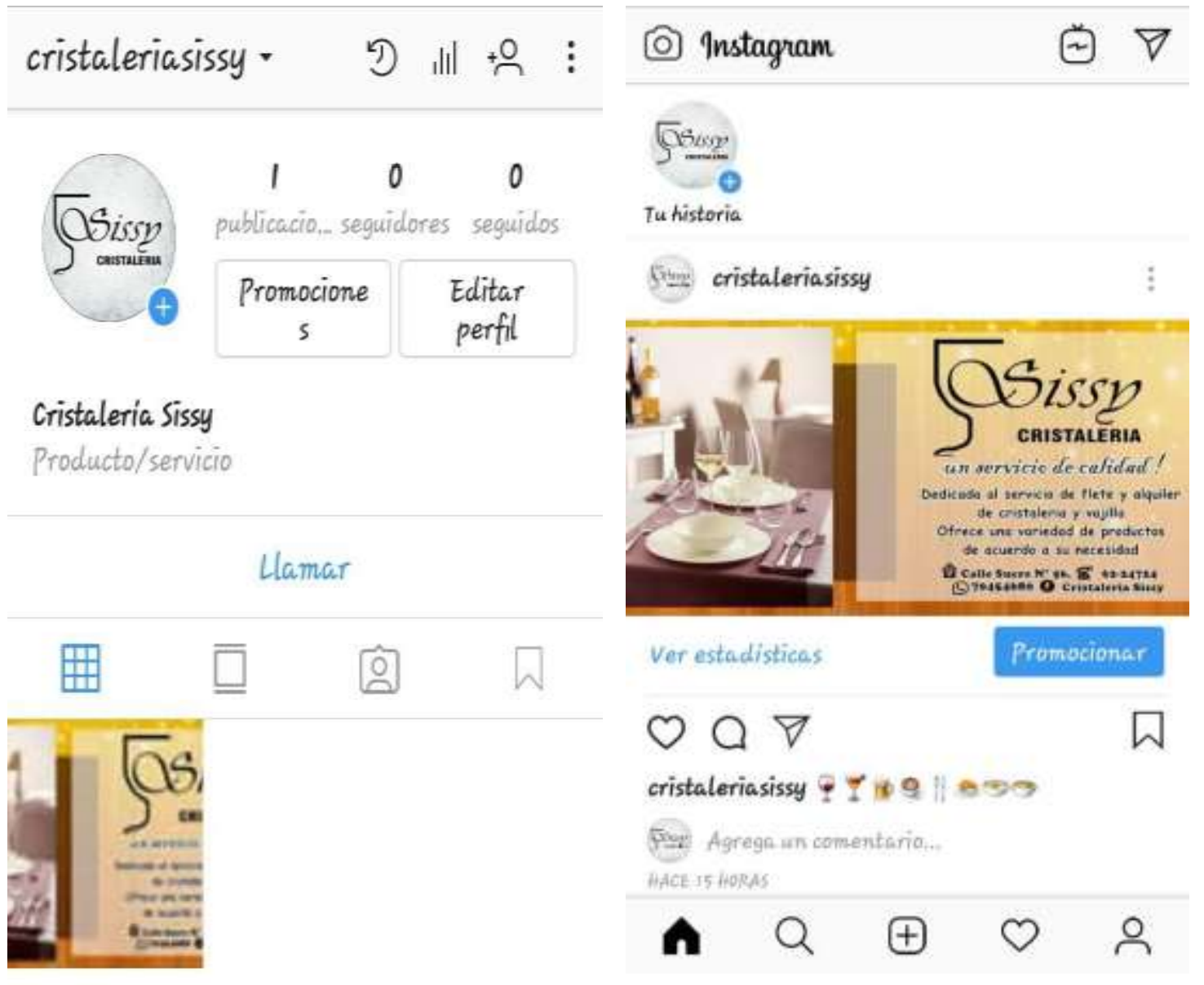

Figura 7. Diseño de página en Instagram "Cristaleríasissy".

(Extraído de https://www.instagram.com/cristaleriasissy/?hl=es-la)

Esta página también permitirá promocionar el servicio. La información de la Cristalería en esta página hace que las personas interesadas puedan llamar $y$ preguntar sobre el servicio que requiere.

\section{- WhatsApp}

Esta aplicación ofrece mensajería y llamadas de una forma simple, segura $y$ confiable, disponible en cualquier teléfono inteligente. La mayoría de la población cuenta con esta aplicación, y según los resultados de la encuesta, el $87 \%$ de la población encuestada mostró interés en conocer la Cristalería "Sissy" dejando su nombre y su número de celular, lo cual permitirá realizar un grupo de WhatsApp y por este medio promocionar el servicio $y$ proporcionar información, anuncios, atender solicitudes, etc. 

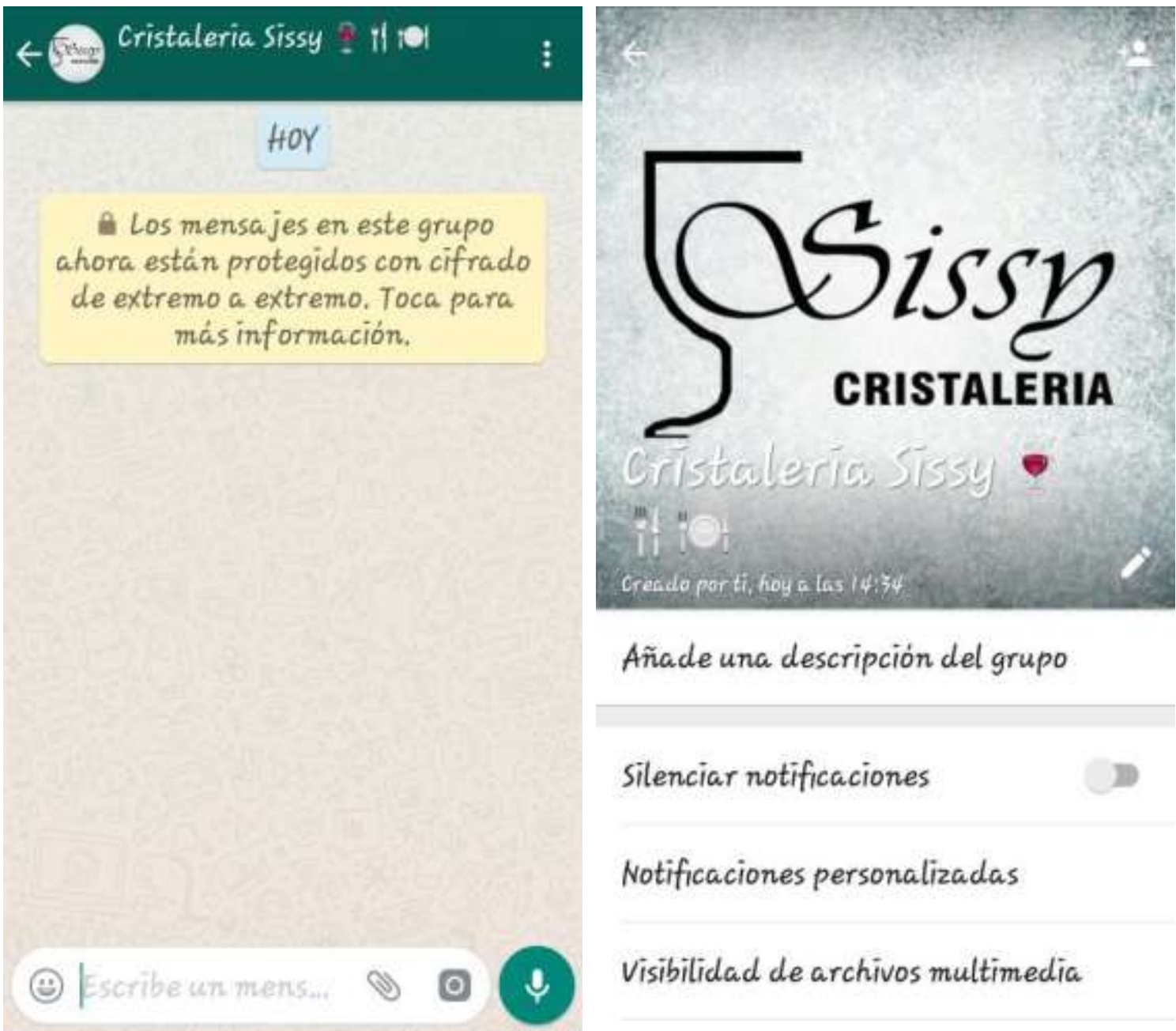

Añade una descripción del grupo

\section{Silenciar notificaciones}

Notificaciones personalizadas

Visibilidad de archivos multimedia

Figura 8. Diseño del grupo de WhatsApp "Cristalería Sissy". Grupo creado por la administradora

\section{Tácticas de relaciones públicas y publicity}

\section{Publicaciones}

Para conseguir la aceptación y confianza del público objetivo, se utilizará material impreso como: tarjetas de presentación, Panfletos y Brochure.
- Tarjetas de presentación

Las tarjetas de presentación se otorgarán a todos los que pasen por la cristalería a preguntar o hacer una consulta.

Dichas tarjetas incluirán el logo de la cristalería y los datos de la empresa, de manera que cuando requieran este servicio puedan contactarnos fácilmente. 
Tabla 7. Presupuesto tarjetas de presentación (expresado en bolivianos)

\begin{tabular}{|c|c|c|}
\hline MES & CANTIDAD & COSTO TOTAL \\
\hline Enero & 1000 & 300 \\
\hline & Costo total tarjetas & 300 \\
\hline
\end{tabular}

Nota: Elaboración propia en base al costo proporcionado por la Imprenta imaGraf

- Panfletos

Durante el año, de vez en cuando, el personal de atención al cliente saldrá a las calles o simplemente fuera de la cristalería para repartir panfletos con la información necesaria de la cristalería.

Tabla 8. Presupuesto panfletos (expresado en bolivianos)

\begin{tabular}{lccc}
\hline \multicolumn{1}{c}{ MES } & CANTIDAD & $\begin{array}{c}\text { COSTO DE IMPRESIÓN } \\
\text { (UNIDAD) }\end{array}$ & COSTO TOTAL \\
\hline Enero & 100 & 1 & 100 \\
Mayo & 100 & 1 & 100 \\
Septiembre & 100 & 1 & 100 \\
\hline & & Costo total panfletos & $\mathbf{3 0 0}$ \\
\hline
\end{tabular}

Nota: Elaboración propia en base a costos estimados

Los panfletos serán impresos, durante los meses establecidos, en la misma cristalería, ya que en la imprenta solo hacen por cantidad.

\section{- Brochure}

Será un gran instrumento de venta para el personal de atención al cliente, así también el cliente tendrá una buena experiencia al conocer $\mathrm{y}$ obtener buena información para luego tomar una decisión.

Será de mucha ayuda en los momentos en el que la Cristalería este lleno de clientes y mientras se le atiende a uno, el resto tendrá a su disposición los brochures, de manera que si tienen alguna consulta o duda sobre los productos o precios puede informarse por medio del brochure, y así tener una mejor información, estos brochures serán propiedad de la cristalería.

Los Brochures serán impresos en la misma cristalería, por el mismo hecho de que en las imprentas realizan trabajos en cantidad, y la Cristalería "Sissy" tendrá solo 3 brochures a disposición. 
Tabla 9. Presupuesto brochures (expresado en bolivianos)

\begin{tabular}{lcccc}
\hline MES & CANTIDAD & $\begin{array}{l}\text { COSTO DE } \\
\text { IMPRESIÓN }\end{array}$ & $\begin{array}{l}\text { COSTO DE } \\
\text { ANILLADO }\end{array}$ & COSTO TOTAL \\
\hline Enero & 3 & 15 & 12 & 27 \\
\hline Costo total panfletos & & & 27 \\
\hline
\end{tabular}

Nota: Elaboración propia en base a costos estimados

\section{Patrocinios}

Se utilizará este instrumento con el fin de apoyar en algunos eventos o acontecimientos de interés general. Ya que las instituciones, como la Alcaldía y Gobernación (clientes de la cristalería), tengan algunos eventos, la Cristalería "Sissy" patrocinará el evento de manera que muchas otras instituciones conocerán el servicio. Así también existen eventos como La Mujer Emprendedora, Fiposí, Feipobol, eventos de moda, etc., será oportunidad para que la cristalería también patrocine estos eventos. Es por eso que la cristalería comprará un banner en el mes de abril para el uso en los diferentes eventos.

Tabla 10. Presupuesto banner (expresado en bolivianos)

\begin{tabular}{cccc}
\hline MES & TIPO DE BANNER & CANTIDAD & COSTO TOTAL \\
\hline Abril & Banner con pedestal & 1 & 200 \\
\hline & & Costo total banner & $\mathbf{2 0 0}$
\end{tabular}

Nota: Elaboración propia en base al costo proporcionado por Gigantografías Pica

\section{Publicity}

Esta herramienta es uno de los más económicos, pues La Cristalería "Sissy", no tendrá que pagar nada, ya que la publicity es una forma de lograr que los medios hagan difusión de la marca de la Cristalería "Sissy" de manera gratuita, y esto se realizará durante los patrocinios que ofrezca la cristalería en ciertos eventos.

Así también al ofrecer un buen servicio al cliente, de alguna forma se puede lograr que estos clientes hagan una difusión de la Cristalería "Sissy", en alguna revista, por ejemplo la Revista Ecos donde publican acontecimientos como Bodas, fiestas de 15 años, graduaciones, cumpleaños, etc. mostrando fotografías de los productos o artículos fletados haciendo mención del nombre de la empresa de manera voluntaria. 


\section{Tácticas de venta personal}

\section{Capacitación a empleados}

Se realizará capacitaciones a los empleados, es decir al personal de atención al cliente, ayudándolos a que se informen y conozcan sobre el posicionamiento, así también, recibirán capacitación de un especialista sobre las etapas de la venta personal, para que de esa manera se pueda tener una comunicación oral e interactiva con el cliente y transmitir la información necesaria. Las capacitaciones se realizarán cada semestre, de la siguiente manera:

\section{Tácticas de marketing directo}

\section{Venta por catálogo}

En este caso las instituciones recibirán un catálogo con los productos y sus características, así también los datos de la cristalería, para el respectivo contacto.

Los catálogos se usaran únicamente para las instituciones que puedan requerir el servicio de flete y alquiler de cristalería y vajilla, ya que muchas veces no saben dónde requerirlo y el catálogo de la cristalería permitirá tener la información y el contacto al instante ante cualquier necesidad lo cual beneficiará en gran manera a las instituciones como también a la cristalería "Sissy".

\section{Cristalería}

Los catálogos serán impresos en la misma cristalería, ya que las instituciones potenciales llegan a un número reducido, y como se dijo anteriormente las imprentas realizan cualquier trabajo en grandes cantidades.

Tabla 11. Presupuesto catálogo (expresado en bolivianos)

\begin{tabular}{lcccc}
\hline MES & CANTIDAD & PAPEL & COSTO DE IMPRESIÓN & COSTO TOTAL \\
\hline Enero & 10 & 100 & 100 & 200 \\
\hline & & Costo total catálogo & 200 \\
\hline
\end{tabular}

Nota: Elaboración propia en base a costos estimados

El personal de atención al cliente realizará las visitas a las diferentes instituciones para ofrecer el servicio y dejar el catálogo para posibles contactos. Esta actividad, se empezará a realizar a partir del mes de febrero en adelante, puesto que el personal recibirá capacitación el mes de enero sobre venta personal, y esto también ayudará a realizar un buen contacto directo, para obtener una respuesta inmediata.

\section{Discusión}

El Marketing tiene un rol muy importante en la economía mundial, porque facilita las relaciones de intercambio entre personas, organizaciones y naciones, por medio de actividades necesarias para que estas puedan tener lo que desean o necesitan. 
Stanton, Etzel y Walker (2007) definen:

El marketing puede producirse en cualquier momento en que una persona o una organización se afanen por intercambiar algo de valor con otra persona $u$ organización. En este sentido amplio, el marketing consta de actividades ideadas para generar $y$ facilitar intercambios con la intención de satisfacer necesidades, o deseos de las personas o las organizaciones. (p.4).

Vale decir que un intercambio es cuando se ofrece algo de valor a un producto o servicio, para cambiarlo por lo que se oferta, es acá donde intervienen los mercadólogos, realizando planes y programas de marketing dirigidas al mercado, compuestas por personas $\mathrm{u}$ organizaciones con necesidades, y los ponen en práctica para estimular y facilitar los intercambios.

\section{Evolución del Marketing}

Desde años remotos, se vio el comercio en los pueblos, llamado "trueque", donde los hombres realizaban transacciones comerciales e intercambiaban productos entre tribus. Actualmente, el comercio ha evolucionado al punto que se realizan transacciones a través de una computadora donde se puede buscar, vender, comprar y adquirir diversos productos o servicios.

Hernández y Maubert (2009) señalan que: "El marketing apareció en el siglo XX, con la intensificación de la competencia, que requería diferentes estrategias para lograr no solamente la diferenciación, sino la ventaja sobre la competencia" (p. 7).
Por lo tanto, a medida que la competencia aumentaba en el mercado, el marketing evolucionaba creando diferentes estrategias para hacer frente a la competencia. Es así que estos autores nos dan a conocer la evolución de la orientación del marketing divididos en seis etapas, a continuación detallaremos en el siguiente cuadro, las características de cada etapa:

En la era de la producción se dio más importancia al productor, y el consumidor no tenía otra alternativa, entonces durante la era del producto las empresas no se daban cuenta que los consumidores compraban los bienes fácilmente, solo se preocupaban en perfeccionar su producto, sin embargo no había ventas, por lo que decidieron hacer énfasis en las ventas, en ese tiempo un vendedor era una profesión muy respetable y lucrativa. Si antes se ponía más énfasis en el producto y el vendedor, en la era del marketing es donde se da más énfasis al consumidor, específicamente a satisfacer sus necesidades, y también, respetar los intereses de la sociedad. Producto de la competencia, el marketing moderno florece, dando lugar a nuevas aplicaciones, con muchas más oportunidades en el mercado.

La evolución que tuvo el marketing a lo largo de estos años, permitió conocer las principales características y el propósito que éste tiene.

En base a estos antecedentes, la competencia en el mercado y las inconstantes necesidades de las personas, muchos autores lograron definir el marketing de manera clara y exacta para una mejor comprensión. 


\section{Definición del marketing}

Kotler y Armstrong (2007) plantean:

En la actualidad, el marketing debe entenderse, no en el sentido arcaico de realizar una venta ("hablar y vender"), sino en el sentido moderno de satisfacer las necesidades del cliente. Si el gerente de marketing entiende bien las necesidades del consumidor; si desarrolla productos que ofrezcan un valor superior; y si fija sus precios, distribuye y promueve de manera eficaz, sus productos se venderán con mucha facilidad. De manera que las ventas y la publicidad son únicamente parte de una "mezcla de marketing" mayor, es decir, del conjunto de herramientas de marketing que funcionan en conjunto para satisfacer las necesidades del cliente y para establecer relaciones con éste. (p. 4).

Por tanto el marketing es un proceso social y administrativo, mediante el cual las personas se relacionan con la empresa la cual satisface sus necesidades y deseos más eficazmente.

Según Monferrer (2013):

En resumen, vemos como la concepción y aplicación del marketing en la empresa ha sufrido una reinterpretación sucesiva del concepto que ha abandonado la mentalidad de hace décadas centrada en la producción y la venta (marketing pasivo o transaccional), para dar cabida a un enfoque más preocupado por la figura del consumidor, sus necesidades $y$ deseos, con el objetivo último de mejorar las relaciones duraderas con estos y el resto de agentes del entorno (marketing activo o relacional). (p.26)
De tal manera que ahora el marketing está dirigido a la satisfacción del cliente, es decir, no solo se centra en los productos, sino que se aplica a todo lo que es susceptible de intercambio entre dos partes ya sea bienes, servicios, ideas, etc., esto hace que el marketing tenga distintas subdisciplinas en función a su aplicabilidad.

\section{Fundamentos teóricos del posicionamiento}

La única manera para que una empresa logre la efectividad del mensaje de su producto o servicio, es siendo selectivo, enfocarse en objetivos precisos, segmentar y sobre todo practicar el posicionamiento. Para entender un poco más acerca de este tema se cita a continuación algunas definiciones de autores que hablan acerca del posicionamiento:

Keller (2008):

...Se refiere a encontrar la "ubicación" adecuada en la mente de un grupo de consumidores o de un segmento de mercado, con el fin de que consideren el producto o servicio de la manera "correcta", y con ello maximizar los potenciales beneficios para la empresa... (p.121).

Fischer y Espejo (2011):

El termino posicionamiento se refiere a un programa general de mercadotecnia que influye en la percepción mental (opiniones, impresiones y asociaciones) que los consumidores tienen de una marca, producto, grupo de productos o empresa, en relación con la competencia... (p.106) 
Ferrell y Hartline (2012):

El posicionamiento, por su parte, se refiere a crear una imagen mental de la oferta de productos y sus características de diferenciación en la mente de los clientes del mercado meta. Esta imagen mental puede basarse en diferencias reales o percibidas entre las ofertas en competencia. (p. 209).

Monferrer (2013):

"En concreto, el posicionamiento de un producto supone la concepción del mismo y de su imagen con el fin de darle un sitio determinado en la mente del consumidor frente a otros productos competidores" (p. 65).

Cervigon (2016):

Es el arte de diseñar la oferta y la imagen de la empresa de modo que ocupen un lugar distintivo en la mente del mercado meta. El posicionamiento es el lugar mental que ocupa la concepción del producto y su imagen cuando se compara con el resto de los productos o marcas competidores, además indica lo que los consumidores piensan sobre las marcas y productos que existen en el mercado. El posicionamiento se utiliza para diferenciar el producto y asociarlo con los atributos deseados por el consumidor. (p. 35).

Todos estos autores, definen al posicionamiento como una percepción mental que los consumidores tienen sobre el producto, servicio, o idea, ocupando un lugar distintivo en su mente de manera que solo el consumidor pueda definirlo como algo correcto o satisfactorio.

Por tanto es de vital importancia estar atentos a los espacios vacíos en la mente del consumidor, y la manera más fácil de realizar esto es siguiendo el consejo que nos dan los siguientes autores.

Ries y Trout (2001) afirman: "Lo primero que el lector necesita para "fijar de manera indeleble su mensaje en la mente" no es en modo alguno un mensaje, sino una mente en blanco en la que no haya hecho mella la marca de alguien más" (p. 22).

Con demasiada información, es difícil que el mensaje llegue a su destino, por lo que es buscar la solución al problema en la mente del cliente o consumidor y dejar una impresión perdurable.

Así mismo Ries y Trout (2001) indican: “...En la Era del posicionamiento, no basta con inventar o descubrir algo, tal vez ni siquiera sea necesario, uno debe ser el primero en llegar a la mente del posible consumidor" (p. 29).

Cabe recalcar que no importa que tan arcaico sea este consejo, para tener éxito en una empresa es necesario ser el primero en ocupar un lugar definitivo y durable en la mente de los consumidores.

\section{Enfoques del posicionamiento}

El concepto de posicionamiento está rebasando fronteras, logrando entrar al terreno corporativo, utilizando como concepto sinónimo de imagen o incluso el de reputación; pero no ha perdido todavía la parte esencial de su sentido original.

Es así que a continuación se presenta dos enfoques que ayudan a clarificar y comprender mejor este concepto, ayudando a reconocer también la importancia que tiene para una empresa. 
El Posicionamiento como Constructo de Emisión Según Capriotti (2009) las organizaciones:

Dibujan, así, un escenario caracterizado por el papel pasivo de los públicos y un papel activo de la organización, que sería la encargada de gestionar los elementos tangibles e intangibles necesarios para lograr una mejor ubicación en la mente de los futuros usuarios o compradores, incidiendo en el papel decisivo que la comunicación tiene en este proceso. (p. 91).

Para algunos autores el posicionamiento se aplica al proceso de enfatizar los atributos distintivos $y$ motivadores de la marca en relación a los competidores; es decir, que posicionar es seleccionar una determinada clientela $y$ decidir cuál va a ser la razón por la que deben preferir nuestro producto o servicio en relación a los competidores.

El Posicionamiento como Constructo de Recepción Al respecto, Capriotti (2009) señala:

Por lo tanto, los autores en este enfoque enfatizan el papel activo de los públicos receptores, tanto en la ubicación del producto/marca/organización en su mente, como en la definición de los atributos ideales en cada caso. Así, el posicionamiento sería una valoración que los sujetos realizan sobre ciertos productos, servicios, marcas u organizaciones a partir de los atributos que mejor definen el mercado en cuestión. (p. 92).

Por tanto, el posicionamiento indica lo que los consumidores piensan de los productos, servicios, marcas, etc. propuestos y actuales en el mercado. Es decir, que es un instrumento de visualización de la realidad del mercado para averiguar la percepción que el público tiene de su mercado quienes aprecian, evalúan y le dan significado. Tipos de posicionamiento

Con el desarrollo de la competencia, el posicionamiento llego a tener un gran alcance en el mercado, por lo cual se vio por conveniente clasificarlo en distintos tipos.

Lo presentado, permite identificar la clase de posicionamiento que una empresa u organización quiere realizar, prácticamente determina la orientación a la que va dirigida de acuerdo a los intereses que ésta tenga.

\section{CONCLUSIONES}

Los hechos que fueron comprobados de toda la información distribuida a lo largo del desarrollo de los capítulos permiten llegar a las siguientes conclusiones.

La sistematización teórica y metodológica sobre el posicionamiento permitió un mejor conocimiento sobre su concepto, procesos, estrategias y la importancia que ésta tiene en cualquier empresa, especialmente en la Cristalería "Sissy".

El diagnóstico del estado actual del posicionamiento efectuado a partir de la aplicación de encuestas y guía de observación al mercado objetivo y entrevistas al personal de la cristalería, permitió conocer el débil posicionamiento que tiene la "Cristalería Sissy" porque no cuenta con criterios eficientes relacionados con la promoción de su servicio para comunicarla en el mercado potosino de esta manera captando nuevos clientes y sobre todo darse a conocer ante su mercado meta en la ciudad de Potosí.

Una vez que se identificó que no existe un buen posicionamiento en la Cristalería "Sissy" se vio por conveniente contribuir con 
herramientas promocionales para el mejoramiento del posicionamiento que beneficie a la empresa así como al mercado objetivo, tales como: Promoción de ventas, Publicidad, Relaciones públicas y publicity, Venta personal y Marketing directo, las cuales forman parte de la estructura de la propuesta.

\section{REFERENCIAS}

Capriotti, P. (2009). Branding Corporativo. Fundamentos para la gestión estratégica de la identidad corporativa. Recuperado de http://www.analisisdemedios.com/brandin g/BrandingCorporativo.pdf

Cervigon, F. J. (s.f). La Mercadotecnia o Marketing Sostenible. Recuperado de https://www.pqs.pe/sites/default/files/201 6/03/aprende-mas/la-mercadotecnia-omarketing-sostenible.pdf

Ferrell, O. C. y Hartline, M. D. (2012). Estrategia de Marketing (5a ed). México: Cengage Learning

Fischer, L. y Espejo, J. (2011). Mercadotecnia (4a ed). México: McGraw-Hill
Hernández, A. y Maubert, C. A. (2009). Fundamentos de Marketing. México: Pearson Educación

Keller, K. L. (2008). Administración estratégica de marca Branding (3a ed). México: Pearson Educación

Kotler, P. y Armstrong, G. (2007). Marketing Versión Latinoamérica (11a ed). México: Pearson Educación

Leal, A. y Quero, M. J. (2011). Manual de marketing y comunicación cultural. Recuperado de http://www.bizkaia.eus/home2/archivo s/DPTO4/Temas/producto44manualdemarketing-y-comunicacion cultural_web.pdf?hash $=898 \mathrm{cdf} 4 \mathrm{dde} 5 \mathrm{fc}$ b436bf5e6b47348d923\&idioma=EU

Monferrer, D. (2013). Fundamentos de Marketing. Recuperado de http://repositori.uji.es/xmlui/bitstream/han dle/10234/49394/s74.pdf

Ries, A. y Trout, J. (2001). Posicionamiento: la batalla por su mente ( $2 \mathrm{a}$ ed). México: McGraw-Hill

Stanton, W. J., Etzel, M. J. y Walker, B. J. (2007). Fundamentos de Marketing (14a ed). México: McGraw-Hill 